\title{
Sonographic septation: a predictor of sequelae of tuberculous pleurisy after treatment
}

\author{
Y-F Lai, ${ }^{1}$ M-C Su, ${ }^{2}$ H-H Weng, ${ }^{3}$ J-T Wu, ${ }^{1}$ C-T Chiu ${ }^{1}$
}

${ }^{1}$ Division of Pulmonary and Critical Care Medicine,

Department of Internal Medicine, E-Da Hospital, I-Shou University, Kaohsiung, Taiwan; ${ }^{2}$ Division of Pulmonary and Critical Care Medicine, Department of Internal Medicine, Chang Gung Memorial Hospital-Kaohsiung, Chang Gung University College of Medicine,

Taiwan; ${ }^{3}$ Department of Diagnostic Radiology, Chang Gung Memorial Hospital-Chiayi Chang Gung University College of Medicine, Taiwan

Correspondence to: Dr C-T Chiu, Division of Pulmonary and Critical Care Medicine, Department of Internal Medicine, E-Da Hospital, 1, Yi-Da Road, Jiau-Shu Tsuen, Yan-Chau Shiang, Kaohsiung, Taiwan; chiuct168@gmail.com

Received 26 October 2008 Accepted 20 May 2009

Published Online First $\mathrm{s}$ June 2009

\section{ABSTRACT}

Background: Findings in the literature have been quite conflicting with respect to predicting residual pleural thickening (RPT) in tuberculous pleurisy (TP). The aim of this study was to determine which sonographic feature of TP might help in predicting the development of RPT.

Methods: Eighty-seven patients with TP were enrolled prospectively. The initial sonographic features were classified as anechoic, homogenously echogenic, complex non-septated and complex septated. The RPT level was measured 12 months after the start of antituberculosis (TB) treatment. Spirometry was performed 6 and 12 months after the start of anti-TB treatment.

Results: A higher odds of an RPT level $>10 \mathrm{~mm}$ was found in patients with positive TB bacillus culture in pleural fluid (OR, 20.9; 95\% Cl, 2.2 to 198.0 ) and a complex septated sonographic pattern (OR, 145.0; 95\% Cl, 22.3 to 942.3). A complex septated sonographic pattern can predict RPT with a sensitivity of $80 \%$, specificity of $96 \%$, positive predictive value of $84 \%$ and negative predictive value of $94 \%$. Patients with an RPT level $>10 \mathrm{~mm}$ had a lower forced vital capacity than those without $(75.4 \%$ (9.2\%) predicted vs $83.2 \%$ (9.5\%) predicted, $p<0.01)$

Conclusion: A complex septated sonographic pattern is a useful sign to predict an RPT level $>10 \mathrm{~mm} 1$ year after the start of anti-TB treatment. An RPT level $>10 \mathrm{~mm}$ is associated with a high probability of decreased lung volumes. Therefore, the initial sonographic feature is beneficial in predicting the sequelae of TP after treatment.

The incidence rate of tuberculosis (TB) in Taiwan was 67.38 per 100000 people in $2006 .{ }^{1}$ Although prevalence and mortality rates have decreased with time owing to effective drug treatment, this infectious disease remains a serious public health issue because of its detrimental complications. Tuberculous pleurisy (TP) is one of the manifestations of $\mathrm{TB}$ and is present in $10 \%$ of all causes of $\mathrm{TB}$ in Taiwan. ${ }^{2}$ Although updated therapeutic regimens can effectively control this infectious disease and minimise its sequelae, residual pleural thickening (RPT) has been found in about half of the patients. ${ }^{3-5}$ We have previously shown that approximately $26 \%$ of patients with TP after treatment are complicated with RPT levels $>10 \mathrm{~mm} .{ }^{6}$ RPT may result in a decreased lung volume and then have important clinical repercussions. It is therefore important to identify the clinical and functional impairment resulting from RPT in patients with TP receiving anti-TB treatment. Thus, the aim of this prospective study was to investigate if sonographic features can serve as a predictor of patient outcomes 12 months after the start of anti-TB drug treatment. To the best of our knowledge, limited studies have been published regarding the evaluation of RPT and the associated impairment in treated TP patients by using the initial songraphic parameters.

\section{METHODS \\ Patients}

Between February 2004 and January 2007, all patients aged 17 years and older with the initial presentation of pleural effusion not treated elsewhere were eligible for this prospective study. Following thoracic sonography, all patients received a needle pleural biopsy and a diagnostic thoracocentesis on the first day of hospitalisation for pleural effusion. Informed consent was obtained from all patients prior to the study. TP was confirmed by the presence of caseating granulomas either with or without acid-fast bacilli on histological examination. Other diseases such as sarcoidosis, rheumatoid arthritis, and fungal or nocardial infection which may produce granulomatous pleurisy were excluded.

Standard anti-TB treatment including isoniazid (300 mg/day), rifampin (450 mg/day in patients $<50 \mathrm{~kg}$ body weight; $600 \mathrm{mg} /$ day in patients $\geqslant 50 \mathrm{~kg}$ body weight), ethambutol (800 mg/day) and pyrazinamide (1500 $\mathrm{mg} /$ day) was given to the patients thereafter. With the exception of pyrazinamide which was administered during the initial 2 months only, all anti-TB drugs were administered for a total of 6 months. No patient had received pleural fluid drainage during the study period.

\section{Chest $\boldsymbol{x}$ rays}

Standard posterior-anterior and lateral chest radiographs of each patient were taken on admission and on a 2-monthly basis during the outpatient follow-up period up to 6 months after completion of the full treatment course. At initial presentation, the amount of pleural effusion on chest $x$ ray was recorded as small (less than one-third of one hemithorax), moderate (between one-third and two-thirds of one hemithorax) or large (more than two-thirds of one hemithorax). Significant RPT was defined as the maximal pleural thickness in excess of $10 \mathrm{~mm}$ on posterior-anterior and lateral chest radiographs. Measurements were made by two independent observers and concurrence was evaluated by using the kappa index.

\section{Pulmonary function tests}

Standard spirometric tests were performed at 6 and 12 months after the beginning of anti-TB treatment (Vitalograph, Spirotrac III, Vitalograph, Lenexa, Kansas, USA). All pulmonary function 

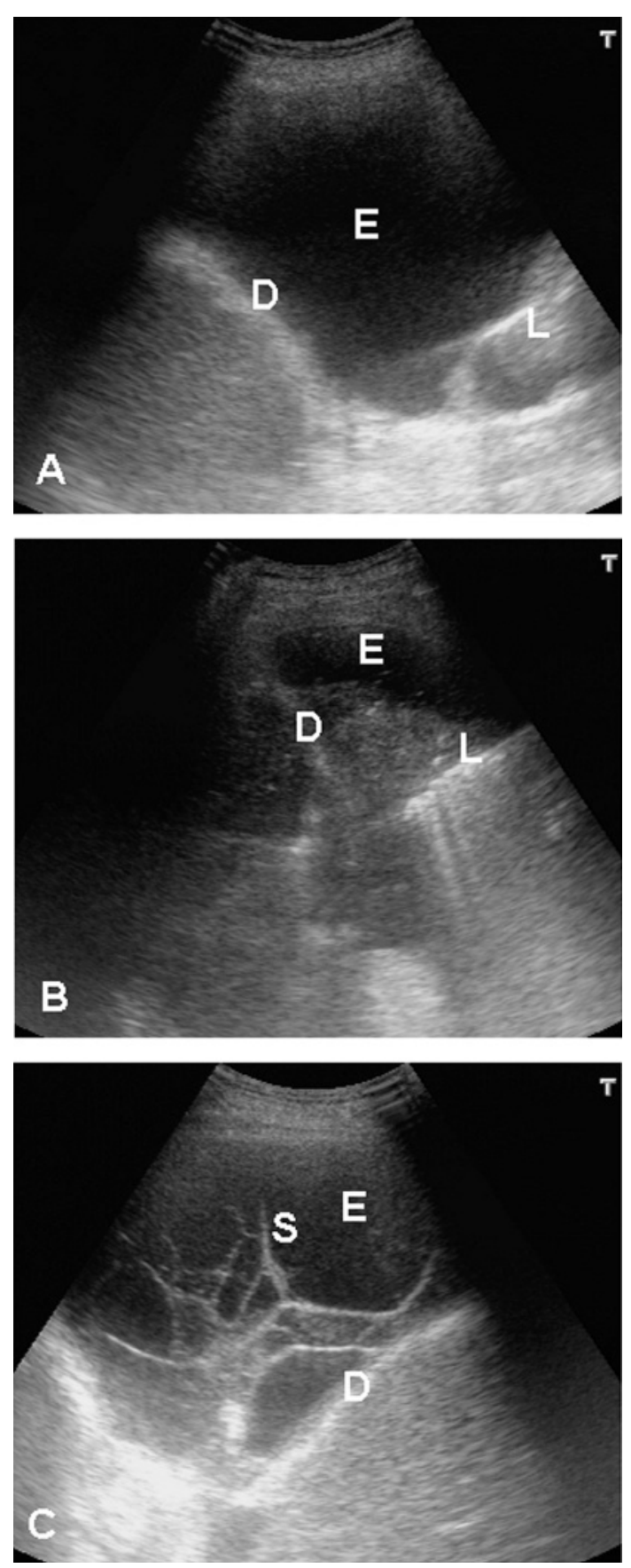

Figure 1 Three representative sonographic patterns of tuberculous pleural effusion. (A) Anechoic; (B) complex non-septated; (C) complex septated. D, diaphragm; E, effusion; L, lung; $S$, septum.

measurements were performed in accordance with the American Thoracic Society guidelines.

\section{Sonography}

All patients were examined with a linear, convex and sector ultrasound transducer with a frequency of 3.5-5.0 $\mathrm{MHz}$ (model SSD 63, Aloka, Tokyo, Japan). According to the internal echogenicity, pleural effusions have been categorised into four types: anechoic, homogenously echogenic, complex non-septated and complex septated. Effusions induced by TB were subclassified as anechoic, complex non-septated or complex septated since the homogenously echogenic type had not been observed among patients with TP. ${ }^{8}$ Effusion was defined as anechogenic if totally echo-free spaces were present between the visceral and parietal pleura (fig 1A). Effusion was defined as complex if echogenic materials were present inside the effusion, and then subclassified into non-septated or septated according to the absence or presence of septa inside the effusions (fig 1B,C).

\section{Statistical analysis}

Data normality was assessed by using the Shapiro-Wilk test. Normally distributed numerical variables are presented as mean (SD), while median values with interquartile range (IOR; $25 \%$ and $75 \%$ ) were expressed for the skewed data. Between-groups comparisons for continuous data were performed using the Student $t$ test or Wilcoxon rank-sum test as appropriate. Independent propotions were compared by using the Fisher exact or $\chi^{2}$ test. We then performed a multiple logistics regression analysis by using a forward stepwise selection procedure to study the independent influences of these variables on the risk of RPT. Results of the logistic regression model were presented as the odds ratio (OR) and 95\% CI. A p value $<0.05$ was considered statistically significant. Statistical analyses were performed using commercially available software (SPSS version 12.0; SPSS, Chicago, Illinois, USA).

\section{RESULTS}

During the 3-year period, 92 consecutive patients with TP were diagnosed. Five were excluded because of non-adherence to the treatment or loss to follow-up. Among the 87 remaining patients, 61 were male, with a mean age of 64.2 (18.2) years. Two experienced readers, who were blinded to the clinical history, assessed the initial size of the pleural effusion and the initial and final pleural thickenings 12 months after the start of anti-TB treatment. The interobserver kappa index was 0.95 , with the strength of agreement being considered very good when $\kappa=0.8-1.0$.

The patient characteristics are shown in table 1 . No pleural thickening was observed in the initial radiographic and sonographic studies. Men were found to be more likely to develop an RPT level $>10 \mathrm{~mm}$ after anti-TB treatment. The pleural fluid analytical variables are listed in table 2 . There was no significant difference in the pleural fluid analysis between the two groups except for a higher lactate dehydrogenase (LDH) level in patient with RPT levels $>10 \mathrm{~mm}$. Table 3 shows the initial sonographic features of all TP patients. No patient presented the feature of a homogenously echogenic pattern.

Table 1 Patient characteristics with RPT $>10 \mathrm{~mm}$ and $\leqslant 10 \mathrm{~mm}$

\begin{tabular}{|c|c|c|c|}
\hline & $\begin{array}{l}\text { RPT > } \\
10 \mathrm{~mm}\end{array}$ & $\begin{array}{l}\text { RPT } \\
\leqslant 10 \mathrm{~mm}\end{array}$ & \\
\hline Characteristics & $(n=20)$ & $(n=67)$ & p Value \\
\hline Male gender $(\%)$ & 90.0 & 64.2 & 0.029 \\
\hline Age (years (SD)) & $61.2(20.3)$ & $65.1(17.6)$ & 0.345 \\
\hline Symptoms to treatment, days & $11(7-30)$ & $10(7-14)$ & 0.620 \\
\hline Smokers (\%) & 55.0 & 34.3 & 0.096 \\
\hline Alcohol abuse (\%) & 15.0 & 9.0 & 0.424 \\
\hline Diabetes mellitus (\%) & 20.0 & 20.9 & 0.931 \\
\hline Pulmonary TB (\%) & 30.0 & 23.9 & 0.581 \\
\hline $\begin{array}{l}\text { Initially large amount of pleural } \\
\text { effusion (\%) }\end{array}$ & 65.0 & 40.3 & 0.148 \\
\hline Loculated pleural effusion (\%) & 20.0 & 16.4 & 0.740 \\
\hline Positive pleural biopsy culture (\%) & 25.0 & 31.3 & 0.782 \\
\hline Positive pleural fluid culture (\%) & 20.0 & 4.5 & 0.046 \\
\hline Sputum positive for AFB (\%) & 25.0 & 16.4 & 0.511 \\
\hline
\end{tabular}

AFB, acid-fast bacillus; RPT, residual pleural thickening; TB, tuberculosis 
Table 2 Pleural fluid findings of patients with RPT $>10 \mathrm{~mm}$ and $\leqslant 10 \mathrm{~mm}$

\begin{tabular}{lcccc}
\hline & $\mathbf{R P T}>\mathbf{1 0} \mathbf{~ m m}$ & & $\mathbf{R P T} \leqslant \mathbf{1 0} \mathbf{~ m m}$ & \\
\cline { 2 - 2 } \cline { 5 - 5 } Pleural fluid analyses & $\mathbf{( n = 2 0 )}$ & & $\mathbf{( n = 6 7 )}$ & $\mathbf{p}$ Value \\
\hline Lymphocyte (\%) & $89.5(76.5-93.5)$ & & $88.0(74.0-93.0)$ & 0.956 \\
Neutrophil (\%) & $10.0(5.0-17.0)$ & & $8.0(3.0-18.0)$ & 0.479 \\
Leucocytes (cells/mm) & $1732(890-2647)$ & & $1960(1000-3520)$ & 0.139 \\
LDH (U/l) & $584(290-682)$ & & $183(127-399)$ & 0.001 \\
Protein (g/dl) & $4.75(4.25-5.45)$ & & $5.0(4.40-5.40)$ & 0.495 \\
Glucose (mg/dl) & $93(43-132)$ & & $95(78-114)$ & 0.684 \\
pH & $7.20(7.18-7.24)$ & & $7.21(7.18-7.29)$ & 0.126 \\
CEA (ng/ml) & $3.0(2.0-3.7)$ & & $3.1(2.4-3.5)$ & 0.571 \\
\hline
\end{tabular}

Data are expressed as median (25-75\% range).

CEA, carcinoembryonic antigen; LDH, lactate dehydrogenase; RPT, residual pleural thickening.

Table 4 demonstrates the initial sonographic features in patients with pure TP (i.e. patients with TP without pulmonary TB). An anechoic pattern on sonography is associated with RPT levels $\leqslant 10 \mathrm{~mm}$ while a complex septated pattern is associated with RPT levels $>10 \mathrm{~mm}$. In univariate analysis, a higher OR of RPT was found in male patients, and in those with positive TB bacillus in pleural fluid culture, higher pleural $\mathrm{LDH}$ and a complex septated echogenic pattern. The results of multivariate logistic regression analysis are presented in table 5 . The association of RPT levels $>10 \mathrm{~mm}$ with positive TB bacillus in pleural fluid culture, and that with a complex septated echogenic pattern remained. In 16 of 20 patients with RPT levels $>10 \mathrm{~mm}$ sonographic examination revealed a complex septated pattern. On the other hand, only 3 of 67 patients with RPT levels $\leqslant 10 \mathrm{~mm}$ had a complex septated pattern during the initial sonographic exam. A complex septated echogenic pattern can predict RPT levels $>10 \mathrm{~mm}$ after 12 months of the start of anti-TB therapy with a sensitivity of $80 \%$, a specificity of $96 \%$, a positive predictive value of $84 \%$ and a negative predictive value of $94 \%$. For patients with pure TP only, a complex septated echogenic pattern can also predict RPT levels $>10 \mathrm{~mm}$ after 12 months of the start of anti-TB therapy with a sensitivity of $79 \%$, a specificity of $96 \%$, a positive predictive value of $85 \%$ and a negative predictive value of $94 \%$.

After 6 months of treatment with anti-TB drugs, all patients were cured according to the clinical response and the improvement of serial chest radiograms. Modification of anti-TB treatment due to drug resistance was not observed. No patient received tube drainage or surgical intervention during the treatment course. At 6 and 12 months after the start of antiTB treatment, patients with RPT levels $>10 \mathrm{~mm}$ had a lower forced vital capacity than those without (73.6\% (9.7\%) predicted vs $81.4 \%(9.6 \%)$ predicted, $p<0.01$; and $75.4 \%$ $(9.2 \%)$ predicted vs $83.2 \%(9.5 \%)$ predicted, $p<0.01$, respectively). In patients with pure $\mathrm{TP}$, forced vital capacity was also lower in those with RPT levels $>10 \mathrm{~mm}$ at 6 and 12 months

Table 3 Initial sonographic findings of patients with tuberculous pleurisy with RPT $>10 \mathrm{~mm}$ and $\leqslant 10 \mathrm{~mm}$

\begin{tabular}{|c|c|c|c|}
\hline & $\begin{array}{l}\text { RPT > } \\
10 \mathrm{~mm}\end{array}$ & $\begin{array}{l}\text { RPT } \\
\leqslant 10 \mathrm{~mm}\end{array}$ & \\
\hline Sonographic features & $(\mathrm{n}=20)$ & $(n=67)$ & p Value \\
\hline Anechoic (\%) & 5.0 & 68.7 & $<0.001$ \\
\hline Complex non-septated echoic (\%) & 15.0 & 26.8 & 0.276 \\
\hline Complex septated echoic (\%) & 80.0 & 4.5 & $<0.001$ \\
\hline
\end{tabular}

RPT, residual pleural thickening.
Table 4 Initial sonographic findings of patients with pure tuberculous pleurisy with RPT $>10 \mathrm{~mm}$ and $\leqslant 10 \mathrm{~mm}$

\begin{tabular}{|c|c|c|c|}
\hline & $\begin{array}{l}\text { RPT > } \\
10 \mathrm{~mm}\end{array}$ & $\begin{array}{l}\text { RPT } \\
\leqslant 10 \mathrm{~mm}\end{array}$ & \\
\hline Sonographic features & $(n=14)$ & $(n=51)$ & p Value \\
\hline Anechoic (\%) & 7.1 & 72.5 & $<0.001$ \\
\hline Complex non-septated echoic (\%) & 21.4 & 21.6 & 1.0 \\
\hline Complex septated echoic (\%) & 71.5 & 5.9 & $<0.001$ \\
\hline
\end{tabular}

after the start of anti-TB treatment $(74.3 \%$ (9.9\%) predicted vs $80.8 \%(9.3 \%)$ predicted, $\mathrm{p}<0.05$; and $75.4 \%(9.9 \%)$ predicted vs $84.0 \%$ (18.4\%) predicted, $\mathrm{p}<0.05$, respectively).

\section{DISCUSSION}

In the present study, we demonstrated the usefulness of sonography in predicting the sequelae of TP after anti-TB treatment. A complex septated pattern shown in the initial sonographic study appears to be a predicting feature for RPT in association with a decreased forced vital capacity.

Dutt et al reported good efficacy of the 6-month regimen of rifampin and isoniazid for TP in 1992, and this regimen has been widely used ever since. ${ }^{9}$ Despite effective anti-TB chemotherapy, RPT is considered to be a common radiographic sequelae in patients with TP after treatment. ${ }^{3-5}$ The incidence of RPT levels $>10 \mathrm{~mm} 1$ year after the start of anti-TB treatment is about $23 \%$ in the present study. RPT may reduce static and dynamic lung volumes and thus have important clinical repercussions. Therefore, clinical and functional impairments induced by RPT are not uncommon in TP patients treated with standard antiTB drugs.

Regarding the development of RPT after anti-TB treatment, most of the studies measured RPT right at the termination of medication (ie, 6 months after the beginning of anti-TB medication). However, further resolutions of RPT after discontinuance of treatment have been reported. ${ }^{10}{ }^{11}$ Radiographic improvements of RPT can occur even at 6 months after the completion of anti-TB chemotherapy. ${ }^{10}{ }^{11}$ Chan et al conducted a retrospective study which assessed 83 patients with TP, 22 of whom had been medically treated 2 years previously. ${ }^{10}$ None of them had significant RPT and their pulmonary function tests did not show any significant impairment when compared with matched control subjects. Han et al reported that the incidence of RPT decreased from $50.6 \%$ at 6 months to $27.1 \%$ at 12 months after the start of medication. ${ }^{11}$ These studies indicate that RPT is a common complication of TP and may regress with time. It is possible that RPT in the initial phase is composed of inflammatory tissues which can be decreased by anti-TB treatment. The local inflammatory hypersensitivity response in TP is mediated in part by a number of proinflammatory mediators such as complement-degrading products, interferon $\gamma$ and cytokines which are abundant in the pleural effusion. ${ }^{10-12}$ As the inflammatory activity was reduced with treatment, the

Table 5 Odds ratio of developing RPT $>10 \mathrm{~mm}$ assessed by multivariate logistic regression analysis

\begin{tabular}{lcc}
\hline Characteristics & Odds ratio $(95 \%$ Cl) & p Value \\
\hline Pleural fluid positive for AFB & $20.9(2.2$ to 198.0$)$ & 0.008 \\
Complex septated echoic & $145.0(22.3$ to 942.3$)$ & $<0.001$ \\
\hline AFB, acidas
\end{tabular}

AFB, acid-fast bacillus; RPT, residual pleural thickening. 
fibrous tissue within the pleural effusion forms RPT which barely changes with time.

In this study we took 12 months after the start of anti-TB chemotherapy as the evaluation point to determine the possible risk factors. Along with demographic and clinical features, serum and pleural fluid analysis, and initial chest radiographic patterns, the initial sonographic features of TP were used to identify which patients were more likely to develop persistent RPT. Findings in the published literature have been quite controversial with respect to the prediction of RPT. ${ }^{32}$ While Barbas et al could not find any association between RPT and demographics or pleural fluid analyses including pleural LDH and glucose levels at the time of initial thoracocentesis, ${ }^{3}$ De Palbo and colleges found that a lower glucose concentration and $\mathrm{pH}$ level in the pleural fluid were associated with RPT. ${ }^{5}$ In the present study the development of RPT does not appear to be well correlated with the initial pleural fluid biochemical analyses except for the level of pleural LDH. Regarding gender difference in RPT, Ruit et al found RPT to be more common in males with TP. ${ }^{13}$ By univariate analysis, we demonstrated a male predominance of RPT. However, this finding appears to be insignificant by multivariate analysis. By multiple logistic regression analysis, we could only identify $\mathrm{TB}$ bacillus in pleural fluid culture and septate complex echo as significant predictors of RPT levels $>10 \mathrm{~mm}$.

The value of sonography for evaluation of pleural disease has been well documented. ${ }^{14}{ }^{15}$ The presence of septation in the pleural fluid revealed by sonography has been shown to be related to the cellular debris in empyema and to clots in the haemothorax. ${ }^{13} 1617$ Sonographic septation is also a useful prognostic indicator of acute thoracic empyema. ${ }^{16}$ Since TP may be a chronic inflammation followed by a pleural fibrosing process which may result in RPT, patients with complex septated echo patterns were more likely to benefit from intrapleural fibrinolytic therapy. ${ }^{16}$ In the present study, 16 of 20 patients with RPT levels $>10 \mathrm{~mm}$ had a septated complex pattern in the initial sonography. On the other hand, there were only 3 out of 67 patients with RPT levels $\leqslant 10 \mathrm{~mm}$ who had the septated complex sonographic patterns. The sensitivity of predicting RPT levels > $10 \mathrm{~mm}$ for a complex septated sonographic pattern is $84 \%$, with a specificity of $96 \%$, positive predictive value of $84 \%$ and negative predictive value of $94 \%$. In patients with pure TB, sonographic septation had a similar predictive value. Therefore, sonographic septation can serve as a useful predictor for the development of RPT after anti-TB treatment.

Akham et al studied 20 patients with TP using ultrasonography, and septations with a lattice-like appearance were found in $6(30 \%)$ patients. ${ }^{17}$ In 1989 Martinez et al reported that winding bands were seen with sonography in 8 out of 21 (38\%) patients with TP. ${ }^{18}$ In the present study we showed that 17 out of $87(21.8 \%)$ patients had septation revealed by thoracic sonography. In a recent study it was shown that early effective drainage with streptokinase for TP may reduce the occurrence of RPT. ${ }^{19}$ Therefore, predicting which patients with TP will develop RPT in the long run is crucial. Thus effective drainage with fibrinolytic therapy in addition to standard anti-TB drug treatment should be introduced for such patients.

In the present study, we also showed that patients with RPT levels $>10 \mathrm{~mm}$ had a lower forced vital capacity than those without. Although Candela et al showed a weak correlation between the forced vital capacity and the degree of radiographic pleural thickening at the end of follow-up, only $6 \%$ of patients had RPT levels $>10 \mathrm{~mm}$ in their study, which is much lower than in our series $(26 \%){ }^{20}$
There were some limitations to this study. First, we were unable to test pulmonary function prior to the initial presentation of TP. It is possible that the difference in forced vital capacity between the two groups existed before the development of TP. However, it is not feasible to collect details of patients' pulmonary function before they become infected with TB. Furthermore, since patients with TP have the potential to have pulmonary $\mathrm{TB}$ and performing pulmonary function tests might raise concern regarding spreading this infectious disease, we did not check lung function at the very beginning. Another limitation is that pleural thickness was not evaluated at initial presentation of these patients, which may make the correlation of complex TP and RPT weak. However, no obvious pleural thickness had been observed in the initial radiographic studies. In addition, the patients had no previous pleural disease or other major pulmonary disease by history taking.

In conclusion, the results of the present study suggest that severe TP shares some common features with thoracic empyema. A sonographic septated complex echo pattern but not the pleural fluid biochemical analysis is a useful feature to predict RPT levels $>10 \mathrm{~mm} 1$ year after the start of anti-TB drug treatment in patients with TP. An RPT level $>10 \mathrm{~mm}$ was associated with a higher probability of a decreased lung volumes.

\section{Competing interests: None.}

Patient consent: Obtained.

Provenance and peer review: Not commissioned; externally peer reviewed.

\section{REFERENCES}

1. Centers for Disease Control. CDC Annual Report 2008. In: Mobilization plan for halving TB incidence in ten years. Taiwan: Centers for Disease Control, Department of Health, 12-19.

2. Hsu CJ, Bai KJ, Chiang $\mathrm{H}$, et al. Tuberculous pleurisy with effusion. J Formos Med Assoc 1999;98:678-82.

3. Barbas CS, Cukier A, Varvalho CR, et al. The relationship between pleural findings and the development of pleural thickening in patients with pleural tuberculosis. Chest 1991;:100:1264-7.

4. Wyser C, Walzl G, Smedema JP, et al. Corticosteroids in the treatment of tuberculous pleurisy: a double-blind, placebo-controlled randomized study. Chest 1996;110:333-8.

5. De Pablo A, Villena V, Echave-Sustaeta J, et al. Are pleural fluid parameters related to the development of residual pleural thickening in tuberculosis? Chest 1997;112:1293-7

6. Lai YF, Chao TY, Wang YH, et al. Pigtail drainage in the treatment of tuberculous pleural effusion: a randomized study. Thorax 2003;58:149-52.

7. American Thoracic Society. American Thoracic Society statements. Snowbird workshop on standardization of spirometry. Am Rev Respir Dis 1979;119:831-8.

8. Chen HJ, Hsu WH, Tu CY, et al. Sonographic septation in lymphocyte-rich exudative pleural effusions: a useful diagnostic predictor for tuberculosis. J Ultrasound Med 2006;25:857-63.

9. Dutt AK, Moers D, Stead WW. Tuberculous pleural effusion: 6 months therapy with isoniazid and rifampin. Am Rev Respir Dis 1992;145:1429-32.

10. Chan CHS, Arnold M, Chan CY, et al. Clinical and pathological features of tuberculous pleural effusion and its long-term consequences. Respiration 1991;58:171-5.

11. Han DH, Song JW, Chung HS, et al. Resolution of residual pleural disease according to time course in tuberculous pleurisy during and after the termination of antituberculosis medication. Chest 2005;128:3240-5.

12. Ellner JJ, Barnes PF, Walkis RS, et al. The immunology of tuberculous pleurisy. Semin Respir Infect 1988;3:335-42.

13. Ruiz EM, Alvarez JL, Ahijado $\mathrm{CH}$, et al. Prognostic factors of clinical outcome of the tuberculous pleural effusion. Med Clin (Barc) 2005;124:491-3.

14. Yang PC. Ultrasound-guided transthoracic biopsy of the chest. Radiol Clin North Am 2000;38:323-43.

15. Yang PC, Luh KT, Chang DB, et al. Value of sonography in determining the nature of pleural effusion: analysis of 320 cases. AJR Am J Roentgenol 1992;159:29-33.

16. Chen KY, Liaw YS, Wang HC, et al. Sonographic septation: a useful prognostic indicator of acute thoracic empyema. J Ultrasound Med 2000;19:837-43.

17. Akhan 0, Demirkazik FB, Ozmen MN, et al. Tuberculous pleural effusions: ultrasonic diagnosis. J Clin Ultrasound 1992;20:461-5.

18. Martinez OC, Serrano BV, Romero RR. Real-time ultrasound evaluation of tuberculous pleural effusions. J Clin Ultrasound 1989;17:407-10.

19. Chung CL, Chen $\mathrm{CH}$, Yeh $\mathrm{CY}$, et al. Early effective drainage in the treatment of loculated tuberculous pleurisy. Eur Respir J 2008;31:1261-7.

20. Candela A, Andujar J, Hernandez L, et al. Functional sequelae of tuberculous pleurisy in patients correctly treated. Chest 2003;123:1996-2000. 